Revue internationale P.M.E.

Économie et gestion de la petite et moyenne entreprise

\title{
Les modes de coordination au sein de réseaux d'innovation pilotés par des TPE
}

\section{Élodie Gardet et Caroline Mothe}

Volume 24, numéro 1, 2011

URI : https://id.erudit.org/iderudit/1012550ar

DOI : https://doi.org/10.7202/1012550ar

Aller au sommaire du numéro

Éditeur(s)

Presses de l’Université du Québec

ISSN

0776-5436 (imprimé)

1918-9699 (numérique)

Découvrir la revue

Citer cet article

Gardet, É. \& Mothe, C. (2011). Les modes de coordination au sein de réseaux d'innovation pilotés par des TPE. Revue internationale P.M.E., 24(1), 9-42. https://doi.org/10.7202/1012550ar
Résumé de l'article

Les très petites entreprises (TPE) sont très impliquées dans la formation de réseaux d'innovation pour valoriser leurs inventions. Cependant, les études empiriques sur le fonctionnement interne des réseaux interorganisationnels, surtout pour l'innovation, restent encore rares. Le vide est encore plus flagrant lorsqu'il s'agit de réseaux pilotés par des TPE. L’objectif ici est donc de relever les modes de coordination mis en place par les pivots TPE pour coordonner les membres de leur réseau. L’analyse empirique qualitative sur sept réseaux d'innovation centralisés met en lumière les modalités de coordination les plus favorables pour un pivot de petite taille et/ou en situation de dépendance. Les résultats permettent aux TPE d'envisager les modalités de coordination adéquates pour mener à bien leur projet d'innovation, y compris lorsqu'elles se retrouvent en situation de dépendance à l'égard d'autres membres de leur réseau. 


\section{Les modes de coordination au sein de réseaux d'innovation pilotés par des TPE}

Élodie GARDET

Caroline MOTHE

IREGE, Université de Savoie

\section{MOTS CLÉS}

\section{Modes de coordination - Dépendance - TPE - Pivot Réseau d'innovation - Taille}

\section{LES AUTEURES}

ÉLODIE GARDET est maître de conférences à I'IUT QLIO (Qualité, Logistique Industrielle et Organisation) de l'Université de Savoie où elle enseigne principalement la comptabilité et l'entrepreneuriat. Ses recherches au laboratoire IREGE s'intéressent notamment aux modes de coordination instaurés dans les réseaux et les différentes dimensions de l'innovation dans les services et plus précisément dans le secteur bancaire.

CAROLINE MOTHE est professeure des universités à l'Institut de management de l'Université de Savoie où elle enseigne essentiellement en stratégie et en management de l'innovation. Elle dirige le master recherche Décisions et Organisations. S'intéressant aux coopérations interfirmes et aux organisations innovantes, elle coordonne actuellement au sein du laboratoire IREGE plusieurs projets de recherche sur les processus d'innovation intra- et interorganisationnels.

\section{RÉSUMÉ}

Les très petites entreprises (TPE) sont très impliquées dans la formation de réseaux d'innovation pour valoriser leurs inventions. Cependant, les études empiriques sur le fonctionnement interne des réseaux interorganisationnels, surtout pour l'innovation, restent encore rares. Le vide est encore plus flagrant lorsqu'il s'agit de réseaux pilotés par des TPE. L'objectif ici est donc de relever les modes de coordination mis en place par les pivots TPE pour coordonner les membres de leur réseau. L'analyse empirique qualitative sur sept réseaux d'innovation centralisés met en lumière les modalités de coordination les plus favorables pour un pivot de petite taille et/ou en situation de dépendance. Les résultats permettent aux TPE d'envisager les 
modalités de coordination adéquates pour mener à bien leur projet d'innovation, y compris lorsqu'elles se retrouvent en situation de dépendance à l'égard d'autres membres de leur réseau.

\begin{abstract}
Small firms are very involved in the formation of innovation networks to enhance their inventions. However, empirical studies on the internal functioning of interorganizational networks, especially for innovation, remain rare. The absence is even starker when it comes to networks controlled by small firms. The objective here is to identify the modes of coordination established by the small-hub firms to coordinate the members of their network. The qualitative empirical analysis on seven centralized networks highlights the innovation coordination arrangements that are the most favorable for a hub firm that is small and/or in a situation of dependence. The results enable small firms to consider the adequate coordination arrangements to carry out their innovation project, including when they find themselves in a situation of dependence on other members of their network.
\end{abstract}

\title{
RESUMEN
}

Muy pequeña empresas están muy involucrados en la formación de redes de innovación para mostrar sus inventos. Sin embargo, los estudios empíricos sobre el funcionamiento interno de las redes entre organizaciones, especialmente para la innovación, siguen siendo escasas. El vacío es aún más evidente cuando se trata de redes controladas por pequeña empresas. El objetivo aquí es identificar los modos de coordinación establecido por los pequeños pivotes para coordinar los miembros de su red. El análisis cualitativo empírico en siete redes centralizadas de innovación destacan los arreglos de coordinación más favorable para un pivote pequeño $y / o$ in situación de dependencia. Los resultados permiten a pequeña empresas considerar los mecanismos de coordinación adecuados para llevar a cabo un proyecto de innovación, incluso cuando se encuentran en una situación de dependencia de otros miembros de su red.

\section{ZUSAMMENFASSUNG}

Mikrounternehmen (< 10 Mitarbeiter) sind für die Verwertung ihrer Erfindungen stark in die Bildung von Innovationsnetzwerken impliziert. Empirische Studien zur Funktionsweise interorganisationaler Netzwerke, vor allem im Zusammenhang mit der Innovation, sind jedoch noch selten. Dieser Mangel an Studien ist noch ausgeprägter bei Netzwerken die durch Kleinunternehmen gesteuert werden. Ziel der Studie ist demzufolge, die Koordinationsmechanismen der Unternehmen bei der Organisation des Netzwerkes zu identifizieren. Eine qualitative Studie bei sieben verschiedenen Netzwerken bringt die vielversprechendsten Mechanismen im erwähnten Kontext hervor. Die Resultate zeigen Kleinstunternehmen auf, wie sie Innovationsprojekte im Verbund durch angepasste Koordinationsformen erfolgreich realisieren können, selbst wenn sie sich in Abhängigkeit zu anderen Netzwerkpartnern befinden. 


\section{Introduction}

De nombreuses recherches mettent en avant la forte implication des petites et moyennes entreprises (PME) dans les activités d'innovation, ces dernières étant souvent effectuées en collaboration, notamment au sein de réseaux d'innovation (Guedj et Picard, 1994; Héraud et Nanopoulos, 1994 ; Doloreux et Melançon, 2007; Pacitto et Tordjman, 1999). Toutefois, les travaux empiriques se focalisant sur la compréhension du fonctionnement interne de ces réseaux sont rares (Ahuja, 2000; Dhanaraj et Parkhe, 2006), les recherches existantes se concentrant sur leur formation, leur structure ou les facteurs de rupture. Or, ces formes organisationnelles sont particulièrement propices à l'échange d'informations et à la transmission de savoir-faire, ce qui favorise les comportements opportunistes (Goerzen, 2007). Ce type de comportement peut être exacerbé dans le cas où le pivot du réseau d'innovation est une PME/TPE (Fonrouge, 2007), d'autant plus que ces réseaux peuvent inclure des organisations de (très) grande taille ${ }^{1}$. Ce sont ces réseaux d'innovation centralisés pilotés par des TPE (définies comme des entreprises de moins de 20 salariés $)^{2}$ que nous étudions dans la présente recherche. Notre objectif est de relever les modalités de coordination ${ }^{3}$ mises en œuvre par un pivot de petite taille, notamment lorsqu'il est en situation de dépendance.

Si les réseaux interorganisationnels ont fait l'objet de nombreux travaux, aucune recherche n'a porté, à notre connaissance, sur l'analyse d'une pluralité de modes de coordination au sein de réseaux pilotés par les TPE. Or, en 2006, on dénombrait 2488000 TPE en France sur 2617300 entreprises. Plus de $95 \%$ des entreprises en France sont donc des TPE, souvent technologiques (Dodgson, 1994). Notre recherche se distingue des travaux antérieurs par:

- l'analyse de plusieurs modes de coordination: les recherches empiriques se sont, pour l'instant, focalisées sur un, voire deux modes de

1. Larue de Tournemine (1994) et Carré et Levratto (2009) insistent sur les fortes complémentarités stratégiques entre grandes firmes et PME en matière d'innovation.

2. Chiffre de la Direction du commerce, de l'artisanat, des services et des professions libérales pour laquelle les TPE sont des entreprises indépendantes de moins de 20 salariés, définition que nous retenons ici. Pour Marchesnay (2003, p. 111), «le passage de la petite à la toute petite entreprise s'effectue dans une zone imprécise qui se situe entre 10 et $20 »$. Pour Julien (1990), cette catégorisation par le nombre d'employés est réductrice: il conviendrait d'inclure le type de propriété, les objectifs de la firme, son potentiel, son organisation, sa croissance, etc.

3. Il faut distinguer ici les «modes» des «modalités », chaque mode de coordination comportant plusieurs modalités (par exemple, la répartition des résultats est un mode de coordination pouvant revêtir deux modalités: la répartition équitable et la répartition égalitaire). 
coordination. Les recherches théoriques, quant à elles, proposent des cadres conceptuels incluant une variété de modes, mais sont assez complexes, rendant ainsi leur opérationnalisation difficile;

- une analyse fine des modes et modalités de coordination: nous allons plus loin dans la compréhension des «modes» en étudiant les différentes «modalités» de coordination;

- l'identification de l'impact de la (très petite) taille du pivot et, plus généralement, des sources de dépendance du pivot sur la mise en place de telle ou telle modalité de coordination. En effet, l'asymétrie de taille entre les membres affecte le management des relations d'alliance (Vidot-Delerue et Simon, 2005). Nous cherchons ainsi à savoir si les modes de coordination diffèrent selon que le pivot est une petite entreprise ou une grande entreprise.

Dans une première partie, une revue de littérature conduit à une définition du réseau d'innovation, des modes de coordination mis en œuvre par le pivot et de la notion de dépendance ${ }^{4}$. La deuxième partie présente les sept cas. Les cinq modes de coordination au sein de six réseaux conduits par une TPE sont comparés à ceux mis en œuvre par un pivot de grande taille dans un septième cas. Cette synthèse des modes (et modalités) de coordination mis en œuvre en fonction (1) de la dépendance (ou non) du pivot (2) et de sa taille est présentée dans une dernière partie.

\section{Modes de coordination et réseaux d'innovation}

Dans la lignée d'Inkpen et Tsang (2005) et de Dhanaraj et Parkhe (2006), le réseau d'innovation est défini comme un ensemble de relations verticales et horizontales avec des organisations diverses (publiques/privées; partenaires/ prestataires), piloté par un pivot dans le but de valoriser l'invention de ce dernier. Nous nous centrons donc ici sur les réseaux d'innovation centralisés (ou centrés autour du pivot), retenant la notion de pivot (Fréry, 1997) et non celle de courtier (Miles, Snow et Coleman, 1992). Lecocq (1999) fait une distinction entre ces deux notions: le pivot est l'acteur unique de régulation des transactions et assure seul les trois rôles de conception, coordination et contrôle. À l'inverse, lorsque ces trois rôles sont tenus par trois organisations différentes, ils sont appelés courtiers. Le pivot est ici prédéfini : c'est celui qui a déposé le ou les brevets et qui, pour valoriser son invention et en raison de sa petite taille et de ses ressources limitées, s'entoure de membres aux

4. Nous parlerons désormais de «dépendance» pour évoquer le «degré de dépendance du pivot à l'égard d'un membre de son réseau d'innovation». 
ressources et compétences complémentaires (Ingham,1991). Ces TPE pivots ont donc des comportements «offensifs» en matière d'innovation (Ingham, 1991; Bernard et Torre, 1994). Leur objectif, afin que le projet d'innovation puisse conduire à une mise sur le marché de l'invention brevetée, est de réguler les transactions (Fréry, 1997) au sein de leur réseau d'innovation au travers de modes et de modalités de coordination adaptés.

\subsection{Modes de coordination et taille du pivot}

Les modes de coordination sont vus comme des arrangements entre unités économiques qui régissent les manières dont ces unités peuvent coopérer pour, ensemble, développer le projet d'innovation (Grandori et Soda, 1995). Cette définition a l'avantage de se focaliser sur les interactions sur le plan stratégique - et non sur le plan opérationnel (comme la répartition des tâches ou les moyens de communication). Il existe une grande diversité dans la terminologie utilisée, qui crée un certain flou conceptuel et sémantique. Les auteurs parlent, de manière quasi indifférenciée, de mécanismes ou de modes internes, de contrôle, de gouvernance, de coordination ou de décisions. Différentes typologies des modes de coordination coexistent: certaines distinguent les modes formels et informels, celles assurant la régulation de l'échange des dispositifs d'incitation et de sanction, ou (de manière la plus courante) les modes transactionnels des modes relationnels. Nous ne retiendrons pour notre part aucune de ces distinctions, car, comme nous le verrons par la suite, elles sont inadéquates pour analyser les modalités prises pour chaque mode ${ }^{5}$.

Nous étudions ici les cinq modes les plus étudiés dans la littérature quelle que soit la terminologie utilisée pour les caractériser: formalisation de l'échange, mode de résolution de conflits, répartition des résultats, garanties et confiance ${ }^{6}$. Que ce soit de manière formelle ou informelle, les partenaires de relations interorganisationnelles doivent fixer les comportements acceptables, la manière de répartir les résultats, les modes de résolution de conflits, etc. (Poppo et Zenger, 2002). Nous présentons ci-après les cinq

5. Par exemple, la résolution de conflits peut avoir plusieurs modalités, certaines pouvant d'ailleurs être de nature tant formelle qu'informelle: résolution conjointe (informelle), persuasion (informelle), coercition (informelle), sanction (formelle ou informelle, mais en général formelle et prévue dans le contrat) ou appel à un tiers (formelle ou informelle, mais en général formelle et prévue dans le contrat). À cela s'ajoute la complexité que certains modes «informels» peuvent être prévus contractuellement. Nous remercions un évaluateur pour cette remarque.

6. Bien d'autres modes, notamment de nature relationnelle, auraient pu être pris en compte: la culture commune, la réputation, l'insertion dans des réseaux sociaux, etc. Nous remercions un évaluateur anonyme pour cette remarque. 
modes retenus ainsi que, pour chacun d'eux, les modalités qui peuvent être prises ( $c f$. tableau 1), en mettant en avant l'impact de la petite taille du pivot sur ces modes.

Formalisation de l'échange - Les modalités formelles, explicites et écrites, comprennent les procédures normalisées, les rapports techniques, les systèmes de comptabilité analytique, de budget et de planification, et les contrats et accords de confidentialité (Martinez et Jarillo, 1989; Gulati,1995; Das et Teng, 1998). Ces modalités peuvent être complétées ou remplacées par des modalités informelles, implicites ou verbales. Celles-ci regroupent la mise en place d'équipes communes (Grandori et Soda, 1995), de séminaires, de réunions et de transferts de personnel (Martinez et Jarillo, 1989). Ces modalités informelles permettent d'abaisser les coûts de transaction (Gulati, 1995), d'accroître la flexibilité stratégique et de réduire les risques de conflit (Nooteboom, Berger et Noorderhaven, 1997). Cependant, leurs temps de mise en place sont longs (Das et Teng, 1998). Or, dans un réseau d'innovation, tout retard sur le délai de mise sur le marché peut rendre le produit issu du projet d'innovation obsolète.

Une TPE est, en général, plus encastrée dans des réseaux sociaux, tant professionnels que personnels du dirigeant, et essentiellement orientée vers des modes relationnels (Jaouen, 2004, 2006). Les alliances stratégiques de TPE seraient caractérisées par l'absence de formalisation, voire, souvent, le refus du contrat (Jaouen, 2006). Les théories contractuelles (des coûts de transaction, positive de l'agence et des contrats incomplets) envisagent deux types de mesure pour évaluer le degré de formalisation de l'échange: l'existence même d'un contrat ou non, et le nombre de clauses? ${ }^{7}$. Les théories relationnelles envisagent également la nature plus ou moins formelle des échanges (courrier, courriels, comptes rendus de réunions ou communications orales, échanges en personne, appels téléphoniques).

Confiance - La confiance interorganisationnelle ${ }^{8}$ est définie comme une condition psychologique sous-jacente qui peut être la cause ou le résultat d'un comportement (comme l'est la coopération) ou d'un choix (Mothe et Ingham, 2003). Les variations de risque et d'interdépendance peuvent altérer à la fois le niveau et la forme de la confiance (Cullen, Johnson et Sakano, 2000). La confiance est souvent considérée comme ayant une influence directe

7. Les clauses contractuelles définissent les droits et obligations des parties. Sans rentrer dans le détail, notons qu'elles sont multiples et concernent l'organisation du projet, les éléments financiers, les résultats, etc.

8. Nous considérons, dans la lignée de Mesquita (2007), que la confiance interorganisationnelle a un statut propre. Pour une revue de la littérature sur la confiance, cf. Mesquita (2007), Mothe et Ingham (2003) ou Simon (2007). 
sur le succès des partenariats (Morgan et Hunt, 1994). Dans l'environnement incertain d'un projet d'innovation, la confiance permettrait de prédire le comportement des membres (Ring et Van de Ven, 1994). Dans les réseaux d'innovation, les imprévus étant quotidiens, les contrats de coopération y sont nécessairement incomplets (Hart et Holmstrom, 1987). Il existe de multiples degrés de confiance qui s'inscrivent sur un continuum allant de la confiance à la méfiance ${ }^{9}$, extrémités que nous retenons ici pour les modalités de ce mode de coordination informel.

Compte tenu de l'importance des relations informelles pour les TPE, on peut supposer qu'un pivot TPE va avoir tendance à privilégier ce type de relation basée sur la confiance. Reste à savoir si contrat formel et confiance se substituent ou se complètent, les recherches récentes tendant à montrer une complémentarité des deux modes de coordination (p. ex. Poppo et Zenger, 2002; Philippart, 2005).

Répartition des résultats - L'un des éléments essentiels de la coopération est la définition d'une règle de partage des résultats. La répartition équitable des résultats est souvent perçue comme une incitation à l'effort pour les membres du projet et est censée favoriser la performance d'un projet d'innovation (Kabanoff, 1991). À l'inverse, la répartition égalitaire des résultats est entendue dans le sens d'uniformité, d'indifférenciation entre les membres du projet. Les différents membres du réseau d'innovation obtiennent une part équivalente des résultats, quelles que soient les ressources et compétences fournies pour le projet d'innovation (partage en parts égales quels que soient les investissements). Ce type de répartition s'avère risqué lorsque les membres contribuent au projet d'innovation dans des proportions différentes, dans la mesure où il peut conduire à un sentiment d'injustice dans la répartition des résultats. Prévue ex ante ou non (distinction importante au sein de la théorie positive de l'agence), cette répartition peut donc être faite de manière égalitaire ou équitable (selon la théorie des contrats incomplets).

À notre connaissance, aucune recherche n'a mentionné de lien entre la taille du pivot et la répartition des résultats.

Garanties - Ces mécanismes de contrôle pour parer les comportements opportunistes (Fréry, 1997) instituent une protection pour le lésé potentiel en rendant coûteuse la sortie du ou des membres opportunistes. De multiples modes de garantie ont été mis en lumière. Fréry (1997) montre qu'il existe d'autres types d'intégration permettant de garantir la loyauté des membres que l'intégration financière (qui consisterait, par exemple, à prendre des participations financières): logistique (maîtrise du capital circulant d'un membre),

9. Sans même parler de défiance... 
médiatique (promotion d'une marque qui sera spontanément reconnue par tous les clients du réseau) et culturelle (recours à des organisations qui entretiennent une proximité non exclusivement économique avec le pivot). Rubin (1990) propose de recourir à deux types d'otages: la réputation (modalité relationnelle) et/ou les actifs spécifiques (modalité issue de la théorie des coûts de transaction). Les futures opportunités d'affaires sont considérées comme un mode de garantie, car le membre opportuniste verra le nombre de ses futures relations d'affaires diminuer (Wu et Choi, 2004). Brousseau (2000) indique qu'un mode de garantie n'est pas toujours contractualisable, car une autorité judiciaire peut difficilement déterminer si les membres ont correctement exécuté leurs engagements contractuels. Dans le cadre de relations interorganisationnelles, les garanties ne sont pas systématiques. Trois cas de figure peuvent survenir (Brousseau et Fares, 2002): 1) aucun système de garantie n'est institué; 2) l'application est unilatérale, c'est-àdire qu'un agent remet un «otage » à l'un ou plusieurs des partenaires; cet otage deviendra la propriété de l'autre s'il ne respecte pas ses promesses;3) l'application est multilatérale, c'est-à-dire que chaque agent remet un otage à son partenaire afin de créer une dépendance mutuelle. Par ailleurs, les modes de garantie (directs et indirects) ne sont pas mutuellement exclusifs. Il est possible d'en cumuler plusieurs, notamment pour se prémunir contre un risque d'opportunisme particulièrement élevé. Les garanties peuvent donc, d'un côté, être directes - ou à effet immédiat (garanties financières ou sur les actifs spécifiques) et/ou indirectes - ou à effet différé (garanties d'image comme la réputation, les futures opportunités d'affaires ou les intégrations médiatiques et culturelle), de l'autre, être appliquées de manière unilatérale ou multilatérale.

Aucune recherche n'a porté sur le lien explicite entre la petite taille du pivot et le type de garantie.

Résolution de conflits - Mohr et Spekman (1994) relèvent plusieurs stratégies de résolution de conflits - mais se limitent à une analyse dyadique. Or, dans un réseau, il faut considérer l'ensemble des interactions: deux à deux, un versus plusieurs, ou plusieurs versus plusieurs (Gomes-Casseres, 1994). Par exemple, si un conflit émerge entre deux partenaires techniques, il est possible que ce soit un autre membre du réseau qui intervienne pour le résoudre (très probablement le porteur de projet ou «pivot»). Or, ce type de situation n'est pas envisagé dans la littérature. Les modalités de résolution de conflits sont complexes dans le cadre d'un réseau d'innovation; en effet, non seulement un projet d'innovation est par nature incertain et ne permet pas d'avoir une visibilité à moyen terme, mais le degré d'engagement des 
membres est très hétérogène. Aussi toute définition ex ante d'une modalité de résolution de conflits est-elle délicate. À l'instar de Mohr et Spekman (1994), nous retenons cinq modalités de résolution de conflits:

- La résolution conjointe du problème: les différentes parties s'engagent à trouver une solution mutuelle au problème.

- La persuasion: l'une des parties (ou un groupe d'organismes) tente de persuader les autres membres que la solution A ou B est la meilleure pour sortir de la situation de conflit. Dans le cadre de réseaux d'innovation, la persuasion peut être à l'initiative du pivot, mais également des membres du réseau, le pivot se faisant alors persuader par les membres de son réseau. Les tentatives persuasives sont généralement plus constructives que l'utilisation de la coercition (Amason, 1996).

- La coercition: elle permet à un ou plusieurs membres de contraindre les autres à choisir la solution qu'il a retenue pour résoudre le conflit. Mohr et Spekman (1994) distinguent la coercition de la domination. Faute de définition et de distinction précise entre les deux notions, nous ne retenons pas cette modalité «domination».

- La sanction: elle consiste à exclure le membre de la coopération.

- Faire appel à un tiers extérieur au réseau d'innovation pour réaliser un arbitrage (arbitre ou tribunal): cette solution est toutefois jugée comme moins bénéfique à une relation pérenne qu'une résolution de conflits en interne, avec absence de parties prenantes extérieures (Mohr et Spekman, 1994).

Là encore, le lien entre la taille du pivot et les modalités de résolution de conflits mises en œuvre n'a, à notre connaissance, pas été étudié. Le tableau 1 synthétise les modes et modalités de coordination et leur origine théorique. 
TABLEAU 1

Modes et modalités de coordination

des membres d'un réseau d'innovation

\begin{tabular}{|c|c|c|}
\hline $\begin{array}{l}\text { Mode de } \\
\text { coordination }\end{array}$ & Modalités de coordination & $\begin{array}{c}\text { Théorie(s) } \\
\text { sous-jacente(s)* }\end{array}$ \\
\hline $\begin{array}{l}\text { Formalisation } \\
\text { de l'échange }\end{array}$ & $\begin{array}{l}\text { - } \text { existence ou non de contrat } \\
\text { - nombre de clauses } \\
\text { - échanges formels ou } \\
\text { informels }\end{array}$ & $\begin{array}{l}\text { TPA, TCT } \\
\text { TCI } \\
\text { Théorie relationnelle }\end{array}$ \\
\hline Confiance ${ }^{* *}$ & $\begin{array}{l}\text { - confiance } \\
\text { - méfiance }\end{array}$ & $\begin{array}{l}\text { - approche normative } \\
\text { (confiance calculée): } \\
\text { TCT } \\
\text { - } \text { approche relationnelle }\end{array}$ \\
\hline $\begin{array}{l}\text { Répartition } \\
\text { des résultats }\end{array}$ & $\begin{array}{l}\text { - égalitaires } \\
\text { - équitables }\end{array}$ & TCI \\
\hline Garanties & $\begin{array}{l}\text { - pas de garantie } \\
\text { - garanties directes et/ou } \\
\text { - garanties indirectes } \\
\text { - unilatérales vs multilatérales }\end{array}$ & $\begin{array}{l}\text { Théories contractuelles } \\
\text { et relationnelles }\end{array}$ \\
\hline $\begin{array}{l}\text { Résolution } \\
\text { de conflits }\end{array}$ & $\begin{array}{l}\text { - } \text { conjointe (par la discussion) } \\
- \text { persuasion } \\
- \text { coercition } \\
- \text { sanction (révocation, sortie) } \\
-\quad \text { appel à un tiers (arbitre ou } \\
\quad \text { tribunal) }\end{array}$ & $\begin{array}{l}\text { TCI } \\
\text { TPA } \\
\text { TCT (TPA, TCI) } \\
\text { TCT }\end{array}$ \\
\hline $\begin{array}{l}* \text { TPA: théorie po } \\
\text { TCI: théorie des }\end{array}$ & \multicolumn{2}{|c|}{ TCT: théorie des coûts de transaction } \\
\hline $\begin{array}{l}\text { ** Notons le statut } \\
\text { théories contrac } \\
\text { relationnelles. }\end{array}$ & $\begin{array}{l}\text { ifique de la confiance, qui est vue c} \\
\text { es et comme un mode de coordin }\end{array}$ & $\begin{array}{l}\text { me un mode de garantie dans les } \\
\text { on à part entière par les théories }\end{array}$ \\
\hline
\end{tabular}

Ces travaux mettent en exergue l'importance des modes de coordination pour appréhender le fonctionnement interne des relations interorganisationnelles et, plus précisément, des réseaux d'innovation. Néanmoins, aucun n'a pris en compte le degré de dépendance du pivot pour comprendre son influence sur chacun de ces modes et modalités. La section suivante recense les principales sources de dépendance du pivot au sein d'un réseau d'innovation. 


\subsection{Impact de la dépendance du pivot sur les modes de coordination}

Toute situation d'interdépendance ou de dépendance mutuelle (Mohr et Spekman, 1994) des partenaires entraîne la manifestation de relations de pouvoir dans les relations interorganisationnelles. Dans un réseau d'innovation où coexistent des entités interdépendantes conservant leur autonomie, la dépendance se réfère au besoin du pivot de maintenir la relation avec tel ou tel membre afin d'atteindre ses objectifs: la mise sur le marché de son projet d'innovation et la réalisation de bénéfices. Le degré d'interdépendance détermine ainsi le degré de pouvoir de chacune des parties dans la relation, les deux étant inversement corrélés (Emerson, 1962). Le rapport de forces initial est déterminé par la taille des partenaires, les ressources et compétences, l'importance stratégique du projet, l'incertitude et l'urgence de la coopération (Tinlot et Mothe, 2005).

La taille - Un déterminant clé des pouvoirs de négociation respectifs des partenaires est lié aux différentiels de taille entre les partenaires. Un partenaire de plus grande taille dispose généralement d'un pouvoir supérieur dans la relation interorganisationnelle (Oliver, 1990). Un réseau associant une firme de taille réduite et une firme plus importante met souvent l'entreprise de petite taille dans une situation de dépendance. Le réseau d'innovation revêt alors pour elle un caractère stratégique plus prononcé que pour la grande entreprise - d'où un déséquilibre des forces en sa défaveur. Une asymétrie au regard de la taille contribuera donc au déséquilibre des pouvoirs de négociation. Toute entreprise de petite taille a besoin des actifs complémentaires de ses partenaires et se trouve en situation de déséquilibre initial. Ainsi, un pivot de petite taille, compte tenu de la difficulté qu'il a à être autosuffisant en ce qui concerne les ressources et compétences pour le projet (Park, Chen et Gallagher, 2002), est souvent en situation de dépendance par rapport aux autres membres de son réseau. Cette dépendance peut n'être que faible si le membre en question est facilement substituable et s'il n'y a pas de situation de «petit nombre» (Williamson, 1985).

Notons que la taille joue ici un double rôle: non seulement elle est une source de dépendance du pivot, qui doit trouver des ressources complémentaires pour mener à bien son projet, mais elle agit aussi comme un facteur indépendant qui influe sur les modalités mises en œuvre par le pivot TPE (cf. section 1.1).

Les ressources et compétences - Le pouvoir de chacun est déterminé par les ressources qu'il amène (Yan et Gray, 1994): physiques, financières, compétences, savoir-faire ou intangibles (réputation d'un partenaire, réseau 
de relations, etc.). La nature des ressources apportées détermine le degré de dépendance d'un partenaire par rapport aux autres. La question des ressources est d'autant plus importante que l'analyse porte sur des réseaux d'innovation asymétriques de type complémentaire, dont la finalité pour le pivot est l'accès à des ressources détenues par le ou les partenaires. C'est le cas notamment dans les réseaux d'innovation. Certaines caractéristiques des ressources tendent à renforcer le pouvoir d'un partenaire: le niveau de spécificité des actifs engagés (Williamson,1985), l'accès à la ressource et son niveau de concentration (Pfeffer et Salancik, 1978) et la valeur intrinsèque des ressources.

L'importance stratégique - Plus la proportion prise par le réseau d'innovation dans le portefeuille d'activités est importante, plus le degré de dépendance de ce même partenaire par rapport aux autres (Thompson,1967; Pfeffer et Salancik, 1978) l'est également - et inversement lié à son niveau de pouvoir. Un réseau d'innovation ayant un caractère stratégique pour le pivot et engageant de façon durable son avenir place celui-ci dans une situation de dépendance face à ses partenaires. L'importance stratégique pour le pivot du projet d'innovation mené au sein d'un réseau est donc fortement corrélée à son degré de dépendance (Yan et Gray, 1994).

L'incertitude - L'équilibre initial des pouvoirs est également lié à l'incertitude eu égard aux partenaires (Crozier et Friedberg, 1977). L'existence d'alternatives pour un partenaire (Bacharach et Lawler, 1980; Yan et Gray, 1994) peut entraîner l'imprévisibilité de son comportement et représente une source majeure de réduction de sa dépendance (Thompson, 1967). Lorsqu'une entreprise est en position de choisir de laisser ou de retirer une ressource vitale pour l'alliance, elle peut exiger des actions de la part de ses partenaires, même si celles-ci sont peu avantageuses pour ces derniers (Harrigan et Newman, 1990).

L'urgence de la coopération - La dimension temporelle explique aussi le pouvoir de l'un des partenaires sur les autres. Le temps est une des dimensions de la marge de manœuvre d'un acteur (Crozier et Friedberg, 1977). La capacité de se fixer un horizon temporel lointain dans une relation de pouvoir est un atout sérieux. Avec un partenaire pressé d'obtenir des résultats, l'indifférence au temps d'un autre confère à ce dernier un avantage indéniable. L'urgence ressentie (en cas de redéploiement stratégique ou d'une opportunité qui ne peut être saisie que par coopération) par l'un des membres du réseau d'innovation le place donc dans une situation défavorable (Harrigan et Newman, 1990). 


\section{FIGURE 1}

Les sources de dépendance et l'impact sur les mécanismes de coordination

\begin{tabular}{l}
\hline Paille \\
\hline Ressources \\
\hline Importance \\
\hline Incertitude \\
\hline Urgence \\
$\rightarrow$ Degré de \\
dépendance \\
Modes de coordination (et modalités associées): \\
- Formalisation de l'échange \\
- Confiance \\
- Répartition des résultats \\
$-\quad$ Garanties \\
- Résolution de conflits
\end{tabular}

Pour se prémunir contre l'opportunisme lié à cette situation de déséquilibre initial, le pivot aura tendance à mettre en place des modes de coordination adéquats. Le degré de dépendance se trouve ainsi cristallisé dans les modes de coordination mis en œuvre au sein du réseau d'innovation par le pivot. Toutefois, aucune recherche ne s'est jusqu'à présent intéressée à l'impact de la dépendance du pivot envers les autres membres de son réseau sur les modes de coordination, et surtout les modalités, mis en place par ce pivot pour mener à bien son projet d'innovation. Par ailleurs, comme indiqué ci-dessus, l'impact de la petite taille du pivot n'a été que peu pris en compte. Nous cherchons donc, à travers une étude exploratoire de sept réseaux d'innovation (six pilotés par des TPE, un par une grande entreprise) de mettre en évidence les modalités de coordination les plus favorables compte tenu de sa petite taille et de son degré de dépendance envers ses partenaires.

\section{2. Étude empirique de sept réseaux d'innovation}

Aucune recherche ne s'est jusqu'à présent penchée sur le lien entre modes de coordination, d'une part, et taille du pivot et dépendance, d'autre part. Cette recherche exploratoire, de nature qualitative, vise à montrer l'impact de ces aspects sur les modes et modalités de coordination mis en place par une TPE pivot du réseau d'innovation. 


\subsection{Méthodologie et terrain}

Nous avons choisi d'étudier des réseaux d'innovation qui, tout en partageant suffisamment de traits communs (innovation technologique, réseaux avec au moins trois membres, etc.), se distinguent par leur taille et leur secteur d'activité ( $c f$. annexe I). Cinquante-sept entretiens ( $c f$. tableau 2) ont été réalisés auprès des membres des réseaux d'innovation: le porteur du projet, les membres financiers, techniques, industriels, commerciaux et juridiques (l'interlocuteur privilégié des pivots pour le projet nous a été indiqué par les pivots en question).

Ces entretiens semi-directifs, d'une durée moyenne de une heure et demie, avaient pour objet de comprendre les modes de coordination à l'œuvre dans ces réseaux. Cette recherche a aussi été réalisée grâce à des données secondaires internes (courriels échangés entre les différents membres du projet, notes internes réalisées par le porteur de projet à l'occasion de la présentation des avancements du projet, plan d'affaires, contrats entre les membres) et externes (Internet, extraits de presse, coupures de journaux). Pour chaque cas, nous avons étudié une diversité de relations, entre les pivots et les membres techniques, financiers, industriels, commerciaux et juridiques (soit une centaine de relations au tota ${ }^{10}$ ).

Nous classons, pour les six réseaux d'innovation pilotés par une petite entreprise, les sources de dépendance en fonction de leur fréquence d'occurrence ${ }^{11}$ :

- La nécessaire acquisition de ressources et compétences est apparue comme la source de dépendance majoritairement rencontrée (cinq cas sur six: A, B, C, E et F). En effet, si le pivot ne possède pas toutes les ressources et compétences indispensables à l'avancement du projet (matériel productif ou canaux de distribution), alors le recours à la coopération est quasiment inévitable et devient une contrainte;

10. Par exemple, dans le projet A, le pivot entretient des relations avec 29 membres techniques: 11 partenaires et 18 prestataires. Le manque de précision quant au nombre de relations étudiées est lié à la complexité de notre objet d'étude: le plus souvent, les acteurs évoquaient un groupe de membres (par exemple, les membres techniques) et non les entreprises de manière individuelle.

11. À l'issue de notre revue de littérature, nous avions retenu quatre sources possibles de dépendance du pivot. Ici, nous n'en expliquons que trois. La taille, élément central pour expliquer la dépendance du pivot, est traitée dans la section 2.3. 


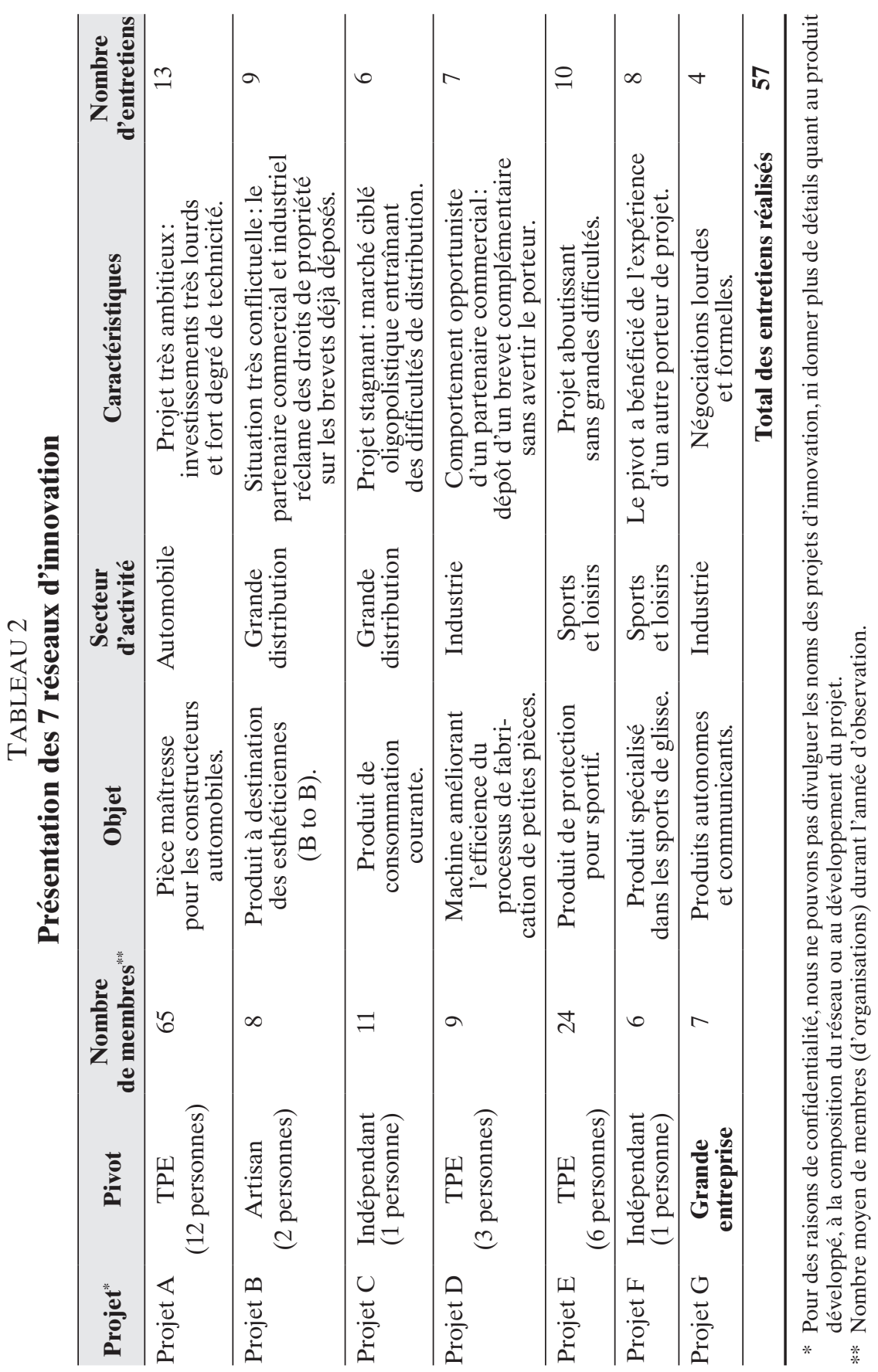


- Trois des six organisations pivots (cas A, C et F) ont été créées pour développer le projet d'innovation. Le projet est donc d'une importance stratégique clé pour ces start-ups, car il en conditionne l'existence même. Si le projet échoue, la start-up disparaît;

- Les pivots $\mathrm{A}, \mathrm{C}$ et $\mathrm{D}$ développent des innovations pour lesquelles de nombreuses technologies substituables peuvent être développées. Ils doivent donc rapidement soumettre leurs innovations au marché, sous peine de voir un autre standard technologique s'imposer.

Dans le cadre d'un réseau d'innovation (Dhanaraj et Parkhe, 2006), le nombre de membres est important. Malgré sa petite taille, le pivot n'est pas en situation de dépendance vis-à-vis de tous.

Le tableau 3 illustre la manière dont nous avons mesuré la dépendance du pivot envers les différents membres de son réseau à partir des différentes sources de dépendance relevées dans la partie théorique ( $c f$. figure 1):

TABLEAU 3

Extrait de la grille de mesure du degré de dépendance du pivot

\begin{tabular}{|c|c|c|c|c|}
\hline & Partenaire 1 & Part. 2 & Part. 3 & Part. $n$ \\
\hline $\begin{array}{l}\text { Taille respective } \\
\text { des partenaires }\end{array}$ & ++ & - & +++ & \\
\hline $\begin{array}{l}\text { Ressources } \\
\text { nécessaires } \\
\text { au projet }\end{array}$ & ++ & - & +++ & \\
\hline $\begin{array}{l}\text { Importance } \\
\text { du projet }\end{array}$ & - & - & - & \\
\hline $\begin{array}{l}\text { Incertitude } \\
\text { du projet }\end{array}$ & + & + & + & \\
\hline $\begin{array}{l}\text { Urgence de } \\
\text { la coopération }\end{array}$ & ++ & - & +++ & \\
\hline $\begin{array}{l}\text { Degré de } \\
\text { dépendance }\end{array}$ & ++ & - & +++ & \\
\hline
\end{tabular}

Nous avons ainsi indiqué, pour chacun des six projets pilotés par une TPE, les membres dont le pivot est dépendant et appréhendé, les raisons du déséquilibre entre les membres - qui a un impact potentiel sur les modalités de coordination mises en œuvre. Nous synthétisons en annexe II l'ensemble des relations pour lesquelles le pivot a été en situation de dépendance. Selon les projets, le pivot n'est pas dépendant des mêmes catégories de membres. 
Nous analysons dans un premier temps les modes de coordination qui varient selon la taille du pivot, dans un second temps, ceux qui varient selon sa plus ou moins forte dépendance.

\subsection{Modes de coordination selon la taille du pivot}

Trois modes de coordination diffèrent selon la taille du pivot: le degré de formalisme, la confiance et la résolution des conflits.

Degré de formalisme - La majorité des pivots TPE (4 sur 6) n'a pris aucun engagement écrit (hormis le contrat de confidentialité) avec les membres qu'ils connaissent et dont ils ne sont pas dépendants (membres facilement substituables). L'absence de contrat permet d'engager la coopération plus rapidement en évitant les coûts et les contraintes juridiques (nombreux allers-retours avec des cabinets juridiques pour rédiger le contrat). Si la confiance se substitue ainsi effectivement, dans certains cas, au contrat, c'est surtout parce que les partenaires en question ne sont pas indispensables. Aussi, en cas de conflit, la sortie de celui-ci sera-t-elle privilégiée, le pivot pouvant aisément, et sans conséquence pour le projet, le «remercier». A contrario, avec les nouveaux membres, les pivots privilégient la signature de contrats, car ils ne peuvent anticiper leurs comportements potentiels. De manière générale, les pivots TPE essaient de réduire le degré de formalisme contractuel (donc le nombre de clauses contractuelles), serait-ce parce qu'ils ne disposent pas des ressources juridiques en interne?

On essaie de ne pas compliquer les choses. Aussi pour des raisons imposées, les petites structures comme $X$ [le pivot] n'ont pas de service juridique, ils n'ont pas d'avocat qui travaille pour eux à temps complet. Notre entreprise, elle, a un service juridique, mais, si l'on commence à les entraîner sur ce terrain-là, on va passer des mois à discuter légal et le projet va être freiné. C'est pour cela que l'on essaie de faire des formats assez simples en termes de contrat quand on travaille avec des sociétés très réactives et à effectif réduit. (Partenaire technique, projet $\mathrm{E}, \mathrm{le}$ 27/07/2006.)

En revanche, le pivot grande entreprise fait signer des contrats de coopération ou de prestation détaillés afin de se protéger au maximum contre d'éventuels comportements opportunistes. Ce contrat fait plus de 200 pages. Les négociations contractuelles sont longues et passent par de nombreuses relectures des différentes versions du contrat par chaque service juridique :

On commence à prendre du retard par rapport aux objectifs techniques. C'est très important d'avoir un accord de consortium qui est en béton, mais c'est au détriment du projet. Il faut bien savoir que l'on ne va pas pouvoir tout mettre dans ce contrat, car il y a beaucoup d'aspects sur lesquels on ne sait pas à quoi 
nous allons aboutir. Je pense que, maintenant, il faut se mettre d'accord sur les droits de propriété et après, il faut foncer. (Partenaire industriel 2, projet G, le 10/01/07.)

Confiance - Dans les cas des réseaux étudiés, le degré de confiance varie en fonction de la taille du pivot et de l'existence (ou non) de relations antérieures réussies. En effet, lorsque les pivots sont des TPE (et, a fortiori, des start-ups créées pour le projet), les partenaires n'ont une confiance $a$ priori que s'ils ont déjà travaillé avec le pivot. En effet, la confiance s'acquiert progressivement par le biais d'interactions, de travail commun, de relations d'échange. Or, la rotation des salariés apparaît comme plus forte dans les grandes entreprises que dans les petites:

Plus vous travaillez avec une personne et plus vous vous connaissez, plus vous savez comment la personne travaille et s'il s'agit d'une personne de confiance. C'est d'ailleurs parce que nous nous connaissons bien que j'ai confiance en $X$ [partenaire PME]. Avec l'entreprise $Y$ [partenaire de moyenne taille], c'est un peu différent: j'ai l'habitude de travailler avec eux et cela s'est toujours bien passé. Mais j'ai toujours une petite appréhension, car, à chaque nouveau projet, on change d'interlocuteur. (Pivot, projet F, le 05/07/06.)

Lors de l'émergence de désaccords, l'utilisation exclusive de la confiance montre ses limites. Il semble préférable de l'associer à un autre mode de coordination:

Avec du recul, je regrette d'avoir fait confiance sans me préoccuper des contrats ou des possibles désaccords, car, lorsque je suis allée voir mon avocate pour tenter de résoudre notre litige, je n'avais que ma parole pour me défendre. C'est sa parole contre la mienne. Donc, pour le juge, c'est très difficile d'arbitrer. (Pivot, projet B, le 26/06/2006.)

Dans le cas du projet $\mathrm{G}$, il existe une confiance a priori entre le pivot et les autres entreprises industrielles - bien qu'ils n'aient jamais travaillé ensemble. Sa réputation, sa légitimité et son image institutionnelle sont le préalable à l'instauration d'une confiance mutuelle.

Résolution des conflits - Ce mode de coordination est le seul parmi les cinq qui soit influencé conjointement par le degré de dépendance et par la taille du pivot ${ }^{12}$. La petite taille d'un pivot implique des structures organisationnelles simples et une souplesse de fonctionnement. En cas de conflit, le pivot TPE peut facilement «sortir» le partenaire: "Comme la rédaction de contrat coûte cher, si l'on ne le fait pas nous-même, on se limite à l'essentiel. En cas de conflit, c'est pareil. Nous pouvons facilement nous séparer d'un partenaire sans avoir à faire aux tribunaux. » (Pivot, projet F, le 01/08/2006.)

12. Nous montrons ici l'influence de la taille, le rôle de la dépendance ayant été traité dans la section précédente. 
A contrario, dans le cas du pivot (de grande taille) de $\mathrm{G}$, le degré de standardisation des tâches est élevé, les procédures et les règles opératoires très formalisées. Les premiers mois du projet $\mathrm{G}$ ont été source de multiples confrontations, notamment entre les trois entreprises industrielles et les laboratoires de recherche. De fréquentes tensions sont apparues, mais celles-ci ont été brèves et n'ont pas remis en cause le projet. Contrairement aux six autres cas, ici, la formalisation est forte et les membres du projet $G$ peuvent difficilement sortir du projet; ils sont dans l'obligation contractuelle de coopérer: "Nous pouvons être sûrs de nous épargner de nous faire la guerre. Dans le contrat, nous avons nommé un arbitre, si vraiment on n'est d'accord sur rien. Mais on essaie d'abord de discuter.» (Pivot, projet G, le 24/11/06.)

Dans le projet $\mathrm{G}$, et dans un premier temps, les membres se sont réunis mensuellement pour discuter des litiges et tenter de trouver des solutions: "Nous avons beaucoup discuté avec les différentes parties prenantes investies dans ce projet. Nous avons multiplié les réunions pour tenter de nous mettre d'accord, mais chacun campait sur sa position.»(Pivot, projet G, le 24/11/06.)

Dans un second temps, faute de consensus, la coercition des trois industriels a été utilisée pour convaincre les laboratoires et résoudre le conflit. Toutefois, si la pression exercée par les industriels n'avait pas fonctionné, ils auraient fait appel à un tiers: «Finalement, nous avons réussi à les faire céder, mais ce n'était pas gagné et nous avions déjà pris contact avec nos services juridiques respectifs pour engager une procédure.» (Pivot, projet G, le 24/11/06.)

\section{TABLEAU 4}

\section{Modes de coordination en fonction de la taille}

\begin{tabular}{lll}
\hline $\begin{array}{c}\text { Modes de } \\
\text { coordination } \\
\text { mis en oeuvre } \\
\text { par le pivot }\end{array}$ & \multicolumn{1}{c}{$\begin{array}{c}\text { Réseau piloté } \\
\text { par une grande } \\
\text { entreprise }\end{array}$} & \multicolumn{1}{c}{$\begin{array}{c}\text { Réseau piloté } \\
\text { par une entreprise } \\
\text { de petite taille }\end{array}$} \\
$\begin{array}{l}\text { Degré de } \\
\text { formalisme }\end{array}$ & $\begin{array}{l}\text { Souvent élevé : contrat lourd } \\
\text { avec de nombreuses clauses, } \\
\text { échanges formels. }\end{array}$ & $\begin{array}{l}\text { Faible: contrat léger, } \\
\text { échanges informels. }\end{array}$ \\
\hline $\begin{array}{l}\text { Degré de } \\
\text { confiance }\end{array}$ & $\begin{array}{l}\text { Forte: une confiance s'est } \\
\text { rapidement formée, le pivot } \\
\text { étant de grande taille et ayant } \\
\text { une réputation. }\end{array}$ & $\begin{array}{l}\text { Faible à forte: la confiance } \\
\text { naît le plus souvent de relations } \\
\text { antérieures (réussies). }\end{array}$ \\
\hline $\begin{array}{l}\text { Résolution } \\
\text { de conflits }\end{array}$ & $\begin{array}{l}\text { Discussion puis coercition ou } \\
\text { appel à un tiers: la sortie de } \\
\text { certains membres entraînerait } \\
\text { des conséquences quant } \\
\text { aux financements publics. }\end{array}$ & $\begin{array}{l}\text { Sortie: le pivot privilégie } \\
\text { la sortie, cette solution étant } \\
\text { plus rapide et moins coûteuse } \\
\text { que le recours à un tiers. }\end{array}$ \\
\hline
\end{tabular}


Le tribunal est une modalité qui est peu utilisée par les pivots TPE, d'une part, parce que la procédure est longue (procédures juridiques pouvant dépasser la durée du projet, car il est possible de renvoyer l'instruction), d'autre part, parce qu'elle est onéreuse (chacune des parties devant payer un avocat pour se faire représenter devant les tribunaux). Le tableau 4 ci-avant synthétise l'influence de la taille sur les modalités de coordination.

\subsection{Modes de coordination selon la dépendance du pivot}

Nos résultats ne mettent en avant que trois modes de coordination variant selon le degré de dépendance du pivot: la répartition des résultats, les garanties et la résolution de conflits.

Répartition des résultats - Dans le cadre de réseaux d'innovation pilotés par un pivot ayant des droits de propriété industrielle (à la suite de dépôts de brevets essentiellement, mais aussi de marque, dessin ou modèle), il reste à prévoir la répartition des résultats financiers potentiels engendrés par la mise sur le marché du projet d'innovation. En général, le pivot propose aux membres une rémunération sous forme de redevances (royalties) récurrentes, à échéances mensuelles ou trimestrielles, le plus souvent calculées en pourcentage du chiffre d'affaires réalisé par le projet. Lorsque le pivot est en situation de dépendance eu égard aux autres membres, la répartition équitable est privilégiée, cette modalité étant estimée comme plus juste. Il y a moins de relations conflictuelles concernant la répartition des résultats avec ce type de répartition:

J'ai apporté une brique indispensable à $X$ [le pivot], donc il me semble normal d'être rémunéré en conséquence. À l'époque, $X$ ne pouvait pas me rémunérer financièrement, c'est pour cette raison que nous avions conclu ce transfert de technologie. (Partenaire commercial, projet D, le 21/09/2006.)

En situation d'absence de dépendance, c'est la répartition égalitaire qui est privilégiée - à condition que le membre ait intégré le projet dès sa genèse et qu'il y participe dans des proportions équivalentes au pivot:

Le projet Jump, c'est un peu comme notre deuxième bébé. On est présent tant pour les bons que pour les mauvais moments. On voit très régulièrement le pivot et c'est ensemble que nous décidons des orientations qui nous semblent les plus favorables au projet. (Partenaire technique, projet F, le 05/07/2006.)

Garanties - Nous avons observé que, plus le pivot est dépendant, plus il a tendance à se prémunir par des garanties directes. La sortie des membres indispensables au projet peut mettre en péril la suite du projet d'innovation. Le pivot prend des garanties directes pour se prémunir contre le risque d'opportunisme et rendre coûteuse la sortie de membres: 
Dans le contrat, nous avons indiqué qu'il devait investir dans des machines spécialisées et que nous le réglerions trois mois après la livraison. Au moins, s'il y a des défauts, on a le temps de les voir et de se retourner. Et il a des pénalités s'il ne nous livre pas en temps et en heure. (Pivot, projet F, le 01/08/2006.)

À l'inverse, moins le pivot est dépendant, moins les garanties directes sont utilisées. En effet, celles-ci sont généralement plus longues à mettre en œuvre (investissement dans des actifs spécifiques, garanties financières nécessitant la signature de contrats) et peu rentables par rapport au risque encouru par le pivot:

On n'a pas de temps à perdre avec des garanties inutiles. On ne va pas perdre du temps à prendre des garanties si on n'a pas de risque dans la relation. C'est comme si vous vous amusiez à assurer une voiture en tous risques alors qu'elle reste dans votre garage toute l'année. Quel est l'intérêt? (Pivot, projet F, le 01/08/2006.)

Par exemple, si le pivot n'est pas en situation de dépendance, il privilégie les garanties indirectes telles que les futures opportunités d'affaires, la notoriété et la crédibilité dans le secteur industriel. Ainsi, dans le cas du projet $\mathrm{D}$, le pivot peut nuire à la réputation du prestataire industriel, car ce dernier travaille sur le même secteur géographique que lui. Le pivot, par sa position centrale au sein du réseau, peut influencer la réputation des membres et, par conséquent, leurs futures opportunités d'affaires.

Résolution de conflits - Lorsque le pivot est dépendant, il n'a pas beaucoup de latitude et les membres influents vont tenter de le persuader de mettre en œuvre telle ou telle solution. En effet, le dépôt de brevet(s) (ou autres titres de propriété industrielle) par le pivot ne lui assure pas une légitimité suffisante: la dépendance du pivot envers les autres membres est élevée, car la faisabilité commerciale n'a pas été démontrée ${ }^{13}$. Le pivot est donc le plus souvent contraint d'accepter les conditions des membres envers lesquels il est dépendant, cette dépendance ne lui permettant pas d'imposer sa solution. Bien qu'il soit à l'origine du projet, il doit se résoudre à accepter les choix des autres membres sous peine de voir le projet échouer:

Si vous avez vraiment besoin de ce partenaire technique et qu'il ne veut pas adopter telle ou telle solution technologique, il y a bien un moment où il faut, soit trouver un autre partenaire qui accepte la solution que vous envisagez, soit

13. Dans les six réseaux pilotés par des petites firmes, le ou les brevets ont été déposés par le pivot (et par le pivot uniquement, ces brevets ne sont pas conjoints) avant la collaboration. C'est donc ce brevet qui est à l'origine du projet d'innovation. L'invention renvoie effectivement à une application industrielle, du moins en théorie, mais elle ne confère pas au pivot une légitimité suffisante pour convaincre les autres membres que le projet d'innovation pourra être mené à bien au plan commercial (nous remercions un évaluateur pour cette remarque). 
Jse résoudre à opter pour la solution qu'il estime être la meilleure. Et, c'est lui l'expert, non? [...] Donc, vous mettez un peu d'eau dans votre vin et essayez. de résoudre cordialement le désaccord, en dialoguant et en faisant en sorte que chacun puisse exposer son point de vue. (Porteur, projet B, le 09/11/2006.)

À l'inverse, lorsque le pivot n'est pas dépendant des membres, la sortie sera privilégiée pour mettre un terme au désaccord. En effet, si les membres sont substituables ${ }^{14}$, le pivot préférera changer rapidement de partenaire avant que d'importants transferts de ressources et compétences ne soient réalisés. En effet, dans ce cas, le pivot a recours à la sanction, qui est un moyen plus rapide que la discussion, cette dernière pouvant engendrer des retards dans l'avancement du projet. La formation d'un nouveau membre n'est pas plus coûteuse que la reconstruction d'une relation détruite par une trahison:

On ne va pas mettre en péril notre projet pour des membres que l'on peut facilement remplacer. Si on passe la moitié de notre temps à essayer de satisfaire tout le monde, notre projet n'avance plus. Donc, maintenant, soit ils sont $O K$ avec notre manière de conduire notre projet, soit ils sortent. On est une petite entreprise et on n'a pas de temps à perdre. (Pivot, projet E, le 05/07/2006.)

Le tableau 5 synthétise l'impact de la dépendance du pivot sur les modalités de coordination.

\section{TABLEAU 5}

\section{Modes de coordination en fonction de la dépendance du pivot}

\section{Modes de coordination mis en oeuvre par le pivot}

\section{Pas de dépendance} du pivot

Égalitaire

Répartition

des résultats
Indirectes

(image et notoriété)

\section{Dépendance}

du pivot
Garanties

Résolution

de conflits
Sortie
Équitable

Directes et indirectes

(futures opportunités d'affaires)

Persuasion du membre dominant

14. Le degré de substituabilité des membres a été évalué de manière subjective dans le guide d'entretien grâce à une question posée au pivot sur la possibilité de changer de partenaire. 
Nos résultats indiquent ainsi les modalités de coordination les plus adaptées ( $c f$. tableaux 4 et 5) compte tenu de la petite taille du pivot et de son degré de dépendance. Le tableau 6 synthétise les modes de coordination qui varient selon ces deux dimensions.

TABLEAU 6

Variables contextuelles clés des cinq modes de coordination

\begin{tabular}{ll}
\hline Modes de coordination & Dimension(s) clé(s) \\
\hline Degré de formalisme & Taille \\
\hline Confiance (ou méfiance) & Taille (si pas de relation antérieure) \\
\hline Répartition des résultats & Degré de dépendance \\
\hline Garanties & Degré de dépendance \\
\hline Résolution de conflits & Degré de dépendance et taille \\
\hline
\end{tabular}

\section{Discussion et conclusion}

Cette recherche vise à améliorer la connaissance du fonctionnement des modes de coordination pouvant être instaurés par un pivot de petite taille d'un réseau d'innovation.

La place de cette firme pivot TPE est ici centrale : c'est autour d'elle que se forme le réseau d'innovation et elle le constitue de manière à mener à bien le projet d'innovation qui vise à transformer l'invention qu'elle a brevetée en un succès commercial. L'impact de la taille de ce pivot est important sur le degré de formalisme (qui est peu élevé, ce qui va dans le sens de Jaouen [2004, 2006]), sur la confiance (la confiance ex ante étant plus forte envers le pivot de grande taille du cas $\mathrm{G}$ que dans les autres cas) et sur la résolution de conflits (où le recours à la sortie du membre est plus difficile lorsque le pivot est de grande taille du fait d'engagements contractuels souvent beaucoup plus forts). Le degré de dépendance de ce pivot, qui diffère selon le type de membre dans ces réseaux multipartites, a également un impact sur trois modes de coordination: la répartition des résultats (équitable en cas de dépendance, égalitaire sinon), les garanties (directes en cas de dépendance et plutôt indirectes sinon) et la résolution de conflits (persuasion en cas de dépendance, sortie du membre sinon). 
Ces résultats sont importants pour les TPE: en effet, contrairement à ce qui est en général avancé sur ce type d'entreprises (notamment sur leur dépendance envers d'autres acteurs), les TPE peuvent non seulement se retrouver à la tête de réseaux d'innovation importants (par le nombre de membres et la nature de ces membres, qui sont parfois des multinationales leaders dans leur domaine d'activité), mais aussi être parfaitement en mesure de les gérer et de les coordonner de manière efficace en mettant en œuvre des modalités appropriées. Aussi les entreprises pourraient-elles envisager le type de coordination à mettre en œuvre selon deux dimensions clés, leur taille, mais surtout leur degré de dépendance à l'égard d'autres membres du réseau d'innovation. En effet, nous montrons que la taille n'a d'influence directe que sur le degré de formalisation des échanges au sein du réseau. Les petites entreprises préfèrent en effet les modes informels aux contrats formels, souvent coûteux à mettre en œuvre. Toutefois, faire reposer une relation au sein d'un réseau d'innovation uniquement sur un mode informel comme la confiance peut susciter des comportements opportunistes de la part des partenaires du pivot. Les trois autres modes de coordination étudiés (répartition des résultats, garanties, résolution de conflits) ne semblent pas dépendre de la taille du pivot, mais de son degré de dépendance. Le choix de la modalité de coordination doit alors se faire en fonction des sources et de l'importance de la dépendance du pivot envers les membres concernés.

D'un point de vue théorique, l'étude des modes et modalités de coordination utilisés par le pivot d'un réseau d'innovation a permis d'affiner les résultats des travaux antérieurs (notamment de Grandori et Soda, 1995; Aggarwal et Hsu, 2009) sur l'influence du degré de dépendance du pivot et de sa taille sur les modalités de coordination utilisées. Ces deux variables de contexte remettent en cause l'univocité de la relation entre modes de coordination et projet d'innovation. Par ailleurs, il ressort de cela que la seule logique de coopération sous-tendue par des modes d'échanges informels ou tacites (Thorelli, 1986; Jarillo, 1988) est peu réaliste. Ce résultat comporte des implications théoriques pour les recherches sur la confiance, qui ont souvent considéré que celle-ci pouvait se substituer aux relations contractuelles. Nos résultats mettent en avant que cette situation est délicate à mettre en œuvre, même lorsque les partenaires se connaissent bien à la suite de relations antérieures satisfaisantes. En effet, les pivots ayant eu uniquement recours à la confiance s'en sont «mordu les doigts », un réseau stable exempt de conflits d'intérêts ou de jeux de pouvoir n'ayant pas été observé. Par exemple, le pivot du projet $\mathrm{D}$ a accordé très facilement sa confiance sans formaliser ses relations d'échange. Il n'a pas évalué le risque que pouvait engendrer cette confiance «quasi aveugle», pensant que les liens sociaux antérieurs lui assuraient la bonne conduite des membres de son réseau, que la confiance agissait comme un mécanisme de contrôle qui minimisait le risque d'une défection 
du partenaire. Il a considéré que les contacts répétés avec son partenaire commercial conduisaient à la production d'une norme de comportement honnête et loyal et d'une volonté de s'impliquer complètement dans le projet Pièces Transfert. Or, son partenaire commercial a profité de la situation en déposant un brevet complémentaire (le pivot avait déposé le brevet sur les liquides et petites pièces et son partenaire l'a déposé sur les poudres).

Nos résultats indiquent donc que, pour un projet d'innovation orienté vers le développement d'un produit, le «contrat relationnel» (MacNeil, 1985; Froehlicher, 1998) n'est pas adapté. Dans les réseaux d'innovation, les contradictions et les divergences d'opinions entre membres sont quasiment inévitables (Miles, Snow et Coleman, 1992), et les conflits d'intérêts ou rivalités de pouvoir empêchent souvent le projet d'évoluer de façon harmonieuse. Dans ces réseaux, dans la lignée de Poppo et Zenger (2002), la complémentarité entre contrat et confiance semble de mise. Le pivot n'a pas eu recours à des contrats, soit lorsqu'il a déjà eu des relations antérieures positives (source de confiance interorganisationnelle), soit lorsqu'il n'est pas en situation de dépendance. Dans ce cas de non-dépendance, le pivot peut aisément «remercier» le partenaire du projet en cas de conflit. L'absence de contrat permet aussi d'engager la coopération plus rapidement en évitant les coûts et les contraintes juridiques, notamment les nombreux allers-retours avec des cabinets juridiques. En situation de dépendance, le pivot a tendance à recourir au contrat - même en cas de relations antérieures réussies - afin de tenter de se prémunir contre l'émergence de conflit.

La présente recherche ne peut être généralisée quant à l'impact de la taille sur les modes de coordination dans la mesure où un seul cas de réseau piloté par une entreprise de grande taille a été analysé. Par ailleurs, des recherches ultérieures devraient prendre en compte le degré anticipé de dépendance, les modes de coordination (comme la formalisation de l'échange, les garanties, la répartition des résultats et, parfois, la résolution de conflit) étant souvent définis a priori. De la même manière, le degré d'asymétrie de la dépendance pourrait faire l'objet de travaux futurs ${ }^{15}$. Nous avons ici traité de l'influence de la dépendance de manière statique; or, ce degré de dépendance évolue au fur et à mesure de l'avancée du projet. Durant les stades amont, le pivot étant une petite entreprise, son degré de dépendance à l'égard des autres membres est souvent élevé; avec l'avancement du projet, le pivot tente de réduire cette dépendance, la situation d'asymétrie initiale pouvant se retourner (Tinlot et Mothe, 2005). Des études longitudinales processuelles (Ring et Van de Ven, 1994) permettraient d'étudier cette évolution de la dépendance du pivot TPE et des modalités de coordination qui en découlent.

15. Nous remercions un évaluateur anonyme de nous avoir suggéré ces deux pistes de recherches. 


\section{ANNEXE I \\ Description de l'échantillon de la recherche}

L'échantillon théorique a été choisi avec les caractéristiques suivantes, selon la revue de littérature effectuée dans la première partie sur les réseaux d'innovation et notre positionnement dans la lignée de Dhanaraj et Parkhe (2006) et Drewello, Soete et Wurzel (2002), notamment:

- Le réseau est dirigé par une entreprise pivot. Que ce soient des réseaux centralisés, comme dans notre cas, ou décentralisés (comme les pôles de compétitivité ou les districts industriels), ils mettent en œuvre des modes de coordination. Dans les réseaux centralisés, le pivot joue le chef d'orchestre et se charge d'organiser au mieux le partage et/ou la mise en commun des ressources des différents membres (Dhanaraj et Parkhe, 2006; Dumoulin, Meschi et Uhlig, 2000). Cette recherche s'intéresse aux modes de coordination mettant en jeu le pivot.

- Le réseau est composé d'au moins trois organisations indépendantes. Les modes de coordination utilisés par un pivot dans une relation bilatérale (soit un projet composé de deux membres) ne permettent pas de comparaison, la validité intra-cas aurait été amoindrie.

- Le projet est basé sur une recherche appliquée. Les recherches fondamentales ne sont pas retenues, car elles ont pour objectif la réalisation de travaux expérimentaux ou théoriques entrepris principalement en vue d'acquérir de nouvelles connaissances. Or, nous étudions des projets dont l'objectif est de mettre un produit sur le marché.

- Le projet d'innovation développe une invention technologique protégée par un brevet, dessin ou modèle (Makadok, 2001). L'innovation technologique est ici retenue pour deux principales raisons. Premièrement, les travaux antérieurs montrent l'importance des réseaux interorganisationnels pour le développement de ce type d'innovation (Langlois et Robertson, 1992). Cette aptitude dépend certes de la R-D interne, mais aussi - et surtout - de la capacité de l'entreprise à intégrer des ressources et compétences externes. Deuxièmement, l'innovation technologique fait l'objet de multiples travaux nous permettant de définir clairement le phénomène. 
Différentes variables contextuelles ont été retenues pour accroître le potentiel de généralisation des résultats:

- Le nombre de membres du réseau: les réseaux étudiés comptent de 4 (projet B) à 55 membres (projet A). Cette variable contextuelle est utilisée dans la plupart des recherches sur les réseaux interorganisationnels (Ahuja, 2000; Goerzen, 2007). Plus ils comportent un nombre important de membres, plus elles paraissent difficiles à gérer et à coordonner (Ethiraj et al., 2005).

- Le secteur d'activité et l'objet sur lequel porte l'innovation: les études de cas réalisées concernent des projets d'innovation dans deux différents secteurs d'activité. Comme la taille du réseau, le secteur d'activité est une variable très utilisée (Meschi, 2006).

- L'étendue géographique du réseau: nombreuses sont les recherches (Fritsch et Lukas, 2001; Suire, 2004) qui insistent sur l'importance de la proximité géographique des organisations d'un réseau pour son bon fonctionnement. Nous vérifions si les membres de chaque réseau d'innovation sont essentiellement locaux, nationaux ou internationaux.

- La taille du pivot et la part de l'effectif investi dans le projet: le nombre total d'employés ainsi que le pourcentage prenant part au projet (Drewello, Soete et Wurzel, 2002) sont des données qui indiquent l'importance du projet pour le pivot.

- Les expériences antérieures de coopération: une entreprise ayant déjà eu des relations de coopération possède une expérience de la gestion d'une telle relation (Nerkar et Roberts, 2004 ; Sorensen et Stuart, 2000).

- Les ressources et les compétences possédées par le pivot: techniques ou commerciales ( $c f$. Callon, Larédo et Mustar, 1995). Le pivot recherche des compétences complémentaires.

- Les recherches empiriques ont pour terrain de prédilection les secteurs de haute technologie: les biotechnologies, les nouvelles technologies et l'automobile (Gilsing et Nooteboom, 2006; Vassolo, Anand et Folta, 2004). Pour autant, les entreprises des autres secteurs sont également innovantes (OSEO, 2004). Pour pallier ce manque, les projets d'innovation étudiés se situent dans des secteurs différents.

Ces caractéristiques correspondent à des facteurs susceptibles d'influencer le fonctionnement des modes de coordination à l'œuvre dans un réseau d'innovation. Le tableau de la page suivante les présente pour les réseaux d'innovation analysés. 


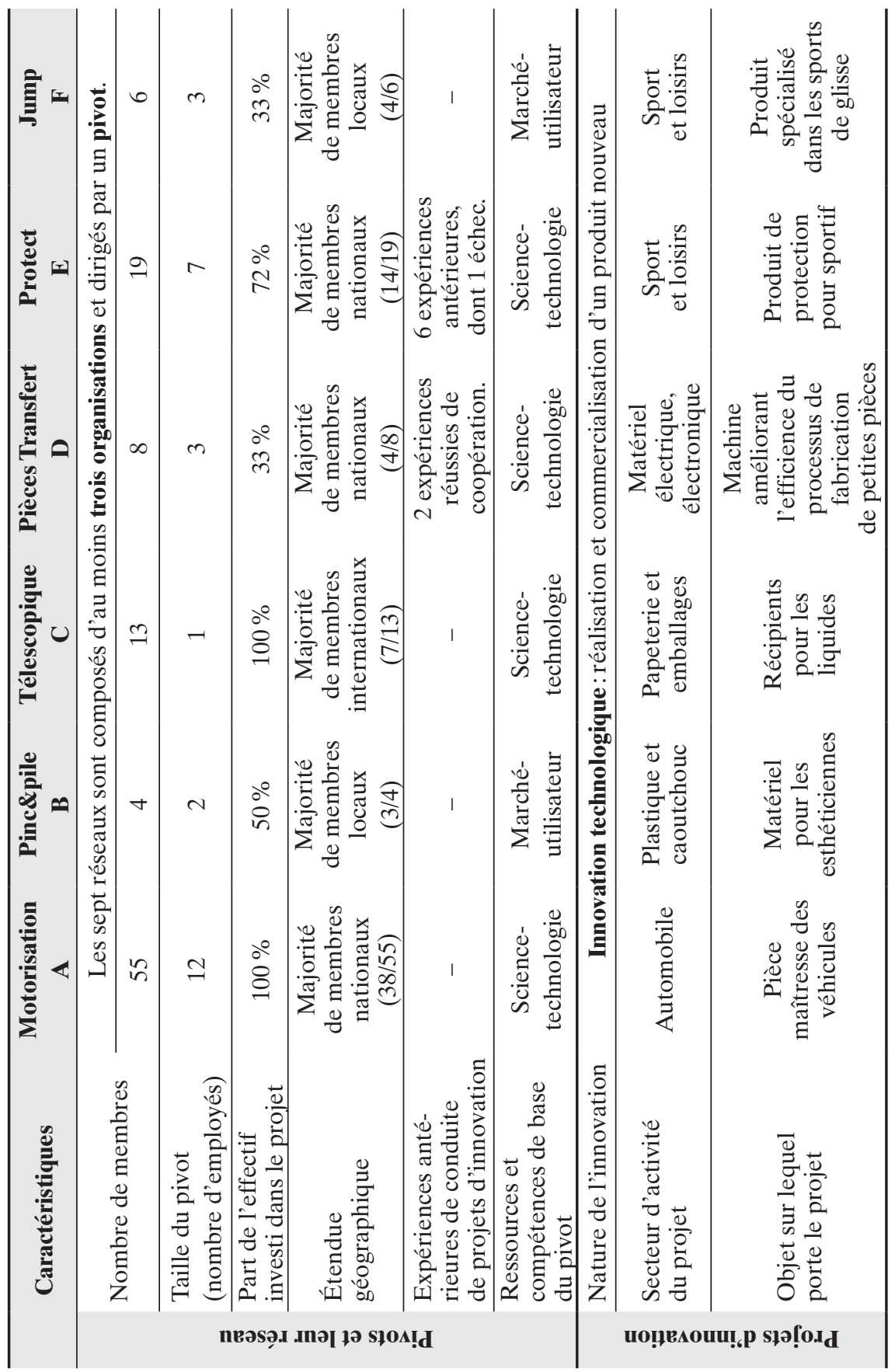


ANNEXE II

Degré de dépendance du pivot TPE par rapport aux membres du projet

\begin{tabular}{lll}
\hline $\begin{array}{l}\text { Projets pilotés } \\
\text { par de petites } \\
\text { entreprises }\end{array}$ & $\begin{array}{l}\text { Pas de dépendance } \\
\text { du pivot envers... }\end{array}$ & $\begin{array}{l}\text { Dépendance } \\
\text { du pivot envers... }\end{array}$ \\
\hline Projet A & $\begin{array}{l}\text { Prestataires techniques } \\
\text { Prestataire juridique }\end{array}$ & $\begin{array}{l}\text { Partenaires et prestataires } \\
\text { financiers } \\
\text { Partenaire industriel } \\
\text { Partenaires techniques }\end{array}$ \\
\hline Projet B & $\begin{array}{l}\text { Prestataire industriel } \\
\text { Partenaire et prestataire } \\
\text { commerciaux }\end{array}$ & $\begin{array}{l}\text { Partenaire juridique } \\
\text { Partenaire technique }\end{array}$ \\
\hline Projet C & $\begin{array}{l}\text { Partenaire juridique } \\
\text { Prestataires techniques } \\
\text { Prestataire industriel }\end{array}$ & $\begin{array}{l}\text { Partenaire commercial } \\
\text { Partenaires techniques } \\
\text { Partenaires et prestataires } \\
\text { financiers }\end{array}$ \\
\hline Projet D & Prestataire industriel & $\begin{array}{l}\text { Partenaire commercial } \\
\text { Partenaires publics financiers }\end{array}$ \\
\hline & Prestataire industriel \\
Projet E & $\begin{array}{l}\text { techniques } \\
\text { Partenaire commercial }\end{array}$ & $\begin{array}{l}\text { Partenaires publics financiers } \\
\text { Partenaires technique } \\
\text { et financier }\end{array}$ \\
\hline
\end{tabular}

* Le partenaire technique correspond au pivot du projet $\mathrm{E}$. 


\section{Bibliographie}

AgGaRWAL,V. et D. HSU (2009), «Modes of cooperative R\&D commercialization by start-ups », Strategic Management Journal, vol. 30, nº 8, p. 835-864.

AHUJA, G. (2000), «Collaboration networks, structural holes, and innovation : a longitudinal study », Administrative Science Quarterly, vol. 45, p. 425-455.

AMASON, A.C. (1996), «Distinguishing the effects of functional and dysfunctional conflict on strategic decision making: resolving a paradox for top management teams », Academy of Management Journal, vol. 39, n 1, p. 123-48.

BACHARACH, S. et E.J. LAWLer (1980), Power and Politics in Organizations, San Francisco, Jossey.

BERNARD, J. et A. TORRE (1994), «Les dynamiques d'innovation et de R\&D des PMI françaises », Revue internationale PME, vol. 7, nos 3-4, p. 19-39.

BROUSSEAU, E. (2000), «What institutions to organize electronic commerce: private institutions and the organization of markets », Economics of Innovation and New Technology, vol. 9, $\mathrm{n}^{\circ}$ 4, p. 245-273.

BROUSSEAU, E. et M. FARES (2002), «Règles de droit et inexécution du contrat: l'apport de la théorie économique des contrats au droit comparé», Revue d'Économie Politique, numéro spécial «L'Économie du droit», sous la dir. de B. Deffains, p. 65-88.

CALLON, M., P. LARÉDO et P. MUSTAR (1995), «Réseaux technico-économiques et analyse des effets structuraux», dans M. Callon et al. (dir.), La gestion stratégique de la recherche et de la technologie, Paris, Economica, p. 415-462.

CARRÉ, D. et N. LEVRATTO (2009), «Place et rôle des PME dans la dynamique de l'innovation », Revue internationale PME, vol. 22, nº 1, p. 47-79.

CROZIER, M. et E. FrIEDBERG (1977), L'acteur et le système, Paris, Seuil.

CUllen, J.B., J.L. JOHNSON et T. SAKANO (2000), «Success through commitment and trust : the soft side of strategic alliance formation », Journal of World Business, vol. 35, p. 223-241.

DAS, T.K. et B.S. TENG (1998), «Between trust and control: developing confidence in partner cooperation in alliances », Academy of Management Review, vol. 23, $\mathrm{n}^{\circ} 3$, p. 491-512.

DHANARAJ, C. et A. PARKHE (2006), «Orchestrating innovation networks », Academy of Management Review, vol. 31, n 3, p. 659-662.

DodGSON, M. (1994), «La stratégie technologique dans les PME: quelques particularités et caractéristiques », Revue internationale PME, vol. 7, nos 3-4, p. 201-218.

DoloreuX, D. et Y. MELANÇON (2007), «Réseaux d'innovation dans les PME en Estrie», Revue internationale PME, vol. 20, n 1, p. 69-90.

DREWELLO, H., B. SOETE et U.G. WURZEL (2002), «Innovation networks in eastern Germany: a still under-utilized potential for human capital formation in the region », Economic Bulletin, vol. 39, n 6, p. 203-208. 
DuMOUlin, R., P.X. MESCHI et T. UHLIG (2000), «Management, contrôle et performance des réseaux d'entreprises: étude empirique de 55 réseaux d'alliances », Finance Contrôle Stratégie, vol. 3, n 2, p. 81-112.

EMERSON, R.M. (1962), «Power-dependence relations», American Sociological Review, vol. 27, n 1, p. 31-41.

ETHIRAJ, S.K., P. KALE, M.S. KRISHNAN et J.V.SINGH (2005), «Where do capabilities come from and how do they matter? A study in the software services industry », Strategic Management Journal, vol. 26, nº 1, p. 25-45.

FONROUGE, C. (2007), «Relations externes et innovation: le cas des biotechnologies», Revue française de gestion, vol. 170, n 1, p. 117-133.

FRÉRY, F. (1997), «Le contrôle des réseaux d'entreprises: pour une extension du concept d'entreprise intégrée», Actes de la Conférence AIMS, Montréal.

FRITSCH, M. et R. LUKAS (2001), «Who cooperates on R\&D? », Research Policy, vol. 30, p. 297-312.

FROEHLICHER, T.(1998), «Les liens sociaux entre dirigeants et le déclenchement de la coopération interentreprises », Finance Contrôle Stratégie, vol. 1, n 1, p. 99-124.

Gilsing, V.A. et B. NoOTEBOOM (2006), «Exploration and exploitation in innovation systems: the case of pharmaceutical biotechnology », Research Policy, vol. $35, \mathrm{n}^{\circ} 1, \mathrm{p} .1-23$.

GOERZEN, A. (2007), «Alliance networks and firm performance: the impact of repeated partnerships », Strategic Management Journal, vol. 28, n 1, p. 487-509.

GOMES-CASSERES, B. (1994), «Group versus group: how alliance networks compete», Harvard Business Review, juillet-août, p. 4-11.

GRANDORI, A. et G. SODA (1995), «Inter-firm networks: antecedents, mechanisms and forms », Organization Studies, vol. 16, $\mathrm{n}^{\text {os }}$ 2, p. 183-214.

GUEDJ, B. et F. PICARD (1994), «Pratiques innovatrices des PME Rhône-alpines: une approche par la diversité des sources de l'innovation technologique», Revue internationale PME, vol. 7, no 3-4, p. 41-64.

GULATI, R. (1995), «Social structure and alliances formation patterns: a longitudinal analysis », Administrative Science Quarterly, vol. 40, p. 619-652.

HARRIGAN, K.R. et W.E. NEWMAN (1990), «Bases of international organization cooperation : propensity, power, persistence », Journal of Management Studies, vol. $27, n^{\circ} 3$, p. 417-434.

HART, O.D. et B. HOLMSTROM (1987), «The theory of contracts», dans T. Bewley (dir.), Advance in Economic Theory, Cambridge, Cambridge University Press.

HÉRAUD, J.A. et C. NANOPOULOS (1994), «Les réseaux de l'innovation dans les PMI: illustration sur le cas de l'Alsace», Revue internationale PME, vol. 7, $\mathrm{n}^{\text {os }} 3-4$, p. 65-86.

INGHAM, M. (1991), «La perception du succès des alliances stratégiques », Revue internationale PME, vol. 4, n 2, p. 43-83. 
INKPEN, A.C. et E.W.K. TSANG (2005), «Social capital, networks, and knowledge transfer », Academy of Management Review, vol. 30, p. 146-165.

JAOUEN,A. (2004), «Les alliances stratégiques: spécificités dans le cas des très petites entreprises », Revue de l'économie méridionale, vol. 52, n 208, p. 263-281.

JAOUEN, A. (2006), «Les stratégies d'alliance des TPE artisanales», Revue internationale PME, vol. 19, $\mathrm{n}^{\text {os }}$ 3-4, p. 111-136.

JARILLO, J.C. (1988), « On strategic networks », Strategic Management Journal, vol. 9 , $\mathrm{n}^{\mathrm{o}}$ 1, p. 31-41.

JULIEN, P.A. (1990), «Vers une typologie multicritères des PME», Revue internationale $P M E$, vol. 3, n ${ }^{\text {os } 3-4, ~ p . ~ 411-425 . ~}$

KABANOFF, B. (1991), «Equity, equality, power and conflict», Academy of Management Review, vol. 16, n' 2, p. 416-441.

LANGLOIS, R.N. et P.L. ROBERTSON (1992), «Networks and innovation in a modular system: lessons from the microcomputer and stereo component industries», Research Policy, vol. 21, nº 4, p. 297-313.

LARUE DE TOURNEMINE, R. (1994), «La complémentarité stratégique entre grande entreprise et PME en matière d'innovation technologique», Revue internationale PME, vol. 7, $\mathrm{n}^{\text {os }} 3-4$, p. 173-200.

LECOCQ, X. (1999), «Le pivot, figure emblématique des réseaux stratégiques », Cahiers du CLAREE, novembre.

MACNEIL, I.R.(1985), «Relational contract: what we do and do not know», Wisconsin Law Review, vol. 3, p. 483-526.

MAKADOK, R. (2001), «Toward a synthesis of the resource-based and dynamiccapability views of rent creation », Strategic Management Journal, vol. 22, n 5, p. 387-401.

MARCHESNAY, M. (2003), «La petite entreprise: sortir de l'ignorance», Revue française de gestion, vol. 3, n 144, p. 107-118.

MARTINEZ, J.I. et J.C. JARILLO (1989), «The evolution of research on coordination mechanisms in multinational corporations », Journal of International Business Studies, vol. 20, nº 3, p. 489-514.

MESCHI, P. (2006), «Réseaux interorganisationnels et survie des alliances », Revue française de gestion, vol. 32, $\mathrm{n}^{\circ}$ 164, p. 33-54.

MESQUITA, L.F. (2007), «Starting over when the bickering never ends: rebuilding aggregate trust among clustered firms through trust facilitators », Academy of Management Review, vol. 32, $\mathrm{n}^{\mathrm{o}}$ 1, p. 72-91.

Miles, R.E., C.C. SNOw et H.J. COLEMAN (1992), «Managing 21st century network organizations », Organizational Dynamics, vol. 20, n 3, p. 5-20.

MOHR, J. et R. SPEKMAN (1994), «Characteristics of partnership success: partnership attributes, communication behavior, and conflict resolution techniques », Strategic Management Journal, vol. 15, n 2, p. 135-152. 
MORGAN, R.M. et S.D. HUNT (1994), «The commitment-trust theory of relationship marketing», Journal of Marketing, vol. 58, n 3, p. 20-38.

MOTHE, C. et M. INGHAM (2003), «La confiance au sein de coopérations: une étude de cas longitudinale», Management International, vol. 7, n 4, p. 17-31.

NERKAR, A. et P.W. ROBERTS (2004), «Technological and product-market experience and the success of new product introductions in the pharmaceutical industry », Strategic Management Journal, vol. 25, p. 779-799.

NoOTEBOOM, B., H. BERGER et N.G. NOORDERHAVEN (1997), «Effects of trust and governance on relational risk », Academy of Management Journal, vol. 40, $\mathrm{n}^{\circ} 2$, p. 308-338.

OLIVER, C. (1990), «Determinants of interorganizational relationships: integration and future directions », Academy of Management Review, vol. 15, nº 2, p. 241-265.

OSEO (2004), Les PME déposantes de brevets en France: caractéristiques et évolutions, Hélène Perrin, Direction des Études et de la Stratégie, Études et bilans réalisés par les experts internes d'OSEO.

PACITTO, J.C. et F. TORDJMAN (1999), «L'innovation technologique dans la très petite entreprise industrielle française: ce que disent les statistiques », Revue internationale PME, vol. 12, n 3, p. 59-90.

PARK, S.H., R. CHEN et S. GALLAGHER (2002), «Firm resources as moderators of the relationship between market growth and strategic alliances in semiconductor start-ups », Academy of Management Journal, vol. 45, n 3, p. 527-545.

PFEFFER, J. et G. SALANCIK (1978), The External Control of Organizations, New York, Harper and Row Publishers.

PHILIPPART, P. (2005), «La dialogique contrat-confiance dans la gestion des alliances interentreprises. Une illustration dans l'industrie automobile», Finance, Contrôle, Stratégie, vol. 8, no 4, p. 177-203.

POPPO, L. et T.R. ZENGER (2002), «Do formal contracts and relational governance function as substitutes or complements?», Strategic Management Journal, vol. 23, no 8, p. 707-725.

RING, P.S. et A.H. VAN DE VEN (1994), «Developmental process of cooperative interorganizational rfelationships », Academy of Management Review, vol. 19, $\mathrm{n}^{\circ} 1$, p. $90-118$.

RuBIN, P.H. (1990), Managing Business Transactions, Controlling the Cost of Coordinating, Communicating and Decision Making, New York, The Free Press.

SiMON, E. (2007), «La confiance dans tous ses états», Revue française de gestion, vol. $33, \mathrm{n}^{\circ} 175$, p. 83-94.

SØRENSEN, J.B. et T.E. STUART (2000), «Aging, obsolescence, and organizational innovation », Administrative Science Quarterly, vol. 45, p. 81-112.

SUIRE, R. (2004), «Des réseaux de l'entrepreneur aux ressorts du créatif: quelles stratégies pour les territoires?», Revue internationale PME, vol. 17, $\mathrm{n}^{\circ} 2$, p. 123-143. 
Thompson, J.D. (1967), Organizations in Action, Social Science Bases of Administrative Theory, New York, Mc Graw Hill.

THORELli, H. (1986), «Networks: between markets and hierarchies», Strategic Management Journal, vol. 7, p. 37-51.

Tinlot, G. et C. MOTHE (2005), «Alliance asymétrique et pouvoir de négociation des partenaires: une relecture de l'alliance Matra-Renault», Management International, vol. 10, $\mathrm{n}^{\circ}$ 1, p. 31-50.

VASSOLO, R.S., J. ANAND et T.B. FOLTA (2004), «Non-additivity in portfolios of exploration activities: a real options-based analysis of equity alliances in biotechnology», Strategic Management Journal, vol. 25, n 11, p. 1045-1061.

VIDOT-DELERUE, H.A. et E. SiMON (2005), «Contrat, confiance et degré d'asymétrie dans les relations d'alliance», Management International, vol. 10, n 1, p. 51-62.

Williamson, O.E. (1985), The Economic Institutions of Capitalism, New York, The Free Press.

WU, W.P. et W.L. CHOI (2004), «Transaction cost, social capital and firms' synergy creation in Chinese business networks: an integrative approach », Asia Pacific Journal of Management, septembre, vol. 21, n 3, p. 325-343.

YAN, A. et B. GRAY (1994), «Bargaining power, management control, and performance in United States - China joint ventures », Academy of Management Journal, vol. 37, p. 1478-1517. 